Supplement of

\title{
Intercomparison and characterization of 23 Aethalometers under laboratory and ambient air conditions: procedures and unit-to-unit variabilities
}

Andrea Cuesta-Mosquera et al.

Correspondence to: Andrea Cuesta-Mosquera (cuesta@tropos.de)

The copyright of individual parts of the supplement might differ from the article licence. 
(a) Ambient air

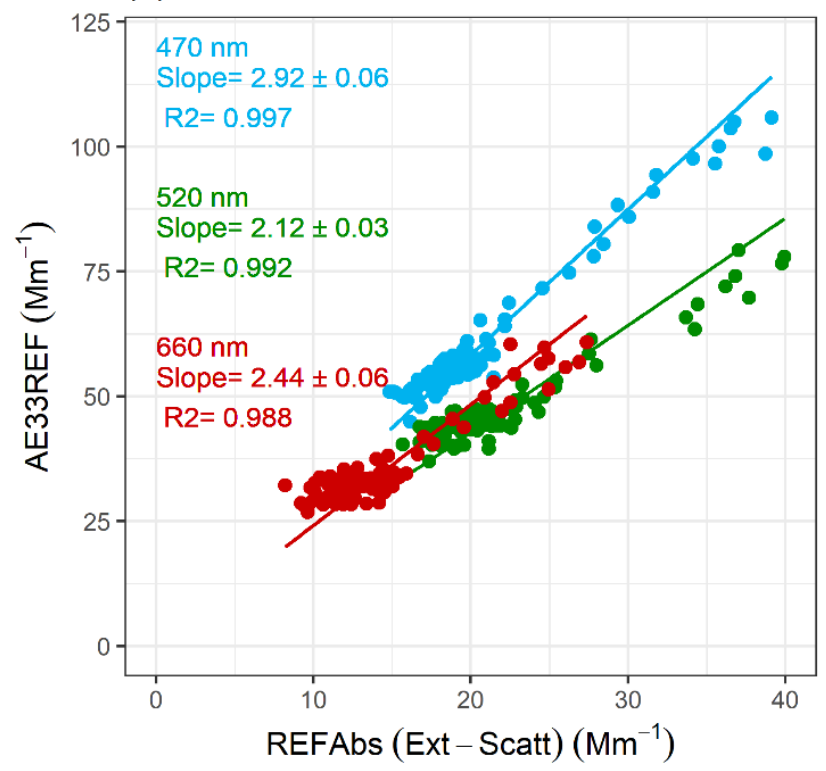

(c) Nigrosin

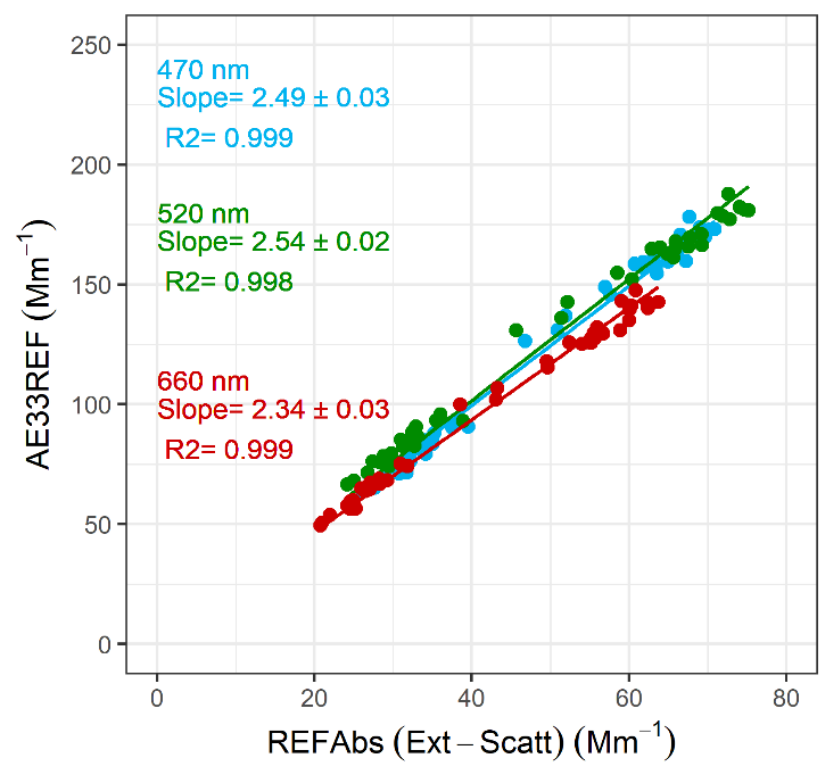

(b) Soot

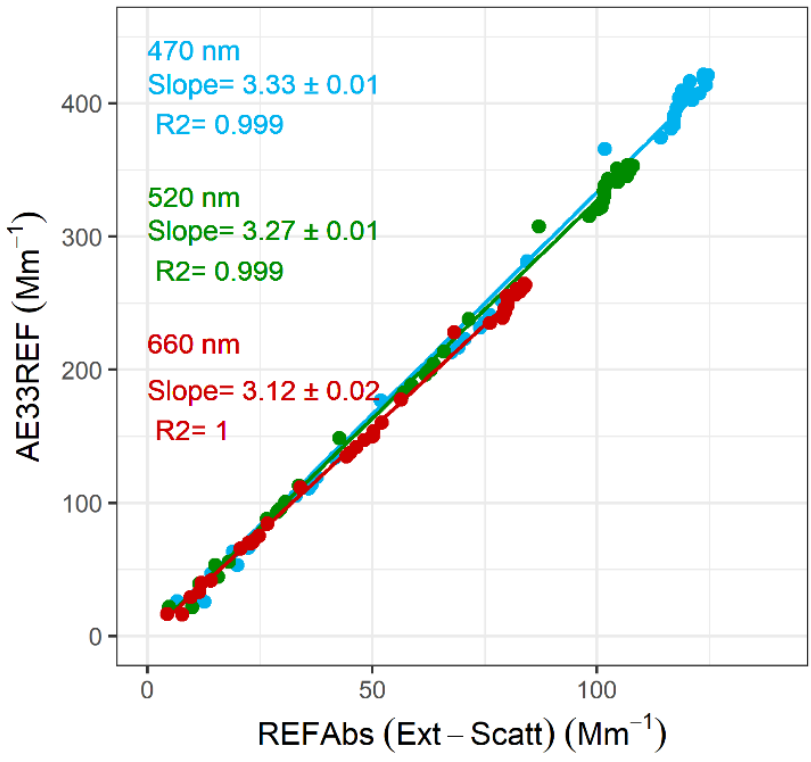

Figure S1. Comparison of absorption reported by the reference AE33 and the reference set-up (Extinction 5 Scattering) of the World Calibration Centre for Aerosol Physics (WCCAP), measuring aerosol samples of (a) ambient air, (b) soot, and (c) nigrosin. The regressions were performed using Deming total least squares and forced through zero; the absorption reported by the reference set-up at $470 \mathrm{~nm}$ and $660 \mathrm{~nm}$ were extrapolated from the original measurement at 450 and $635 \mathrm{~nm}$, to allow comparability with the reference aethalometer. The absorption reported by the reference set-up at $525 \mathrm{~nm}$ was directly compared with the absorption reported by the AE33 REF at

$10520 \mathrm{~nm}$. These wavelengths are not identical, but we consider LEDs have rather wide spectra (Müller et al., 2011). The differences in the slopes are due to different size distributions of sampled particles and due to the wavelength dependent cross-sensitivity to scattering of filter photometers - this is a topic of further investigation. 


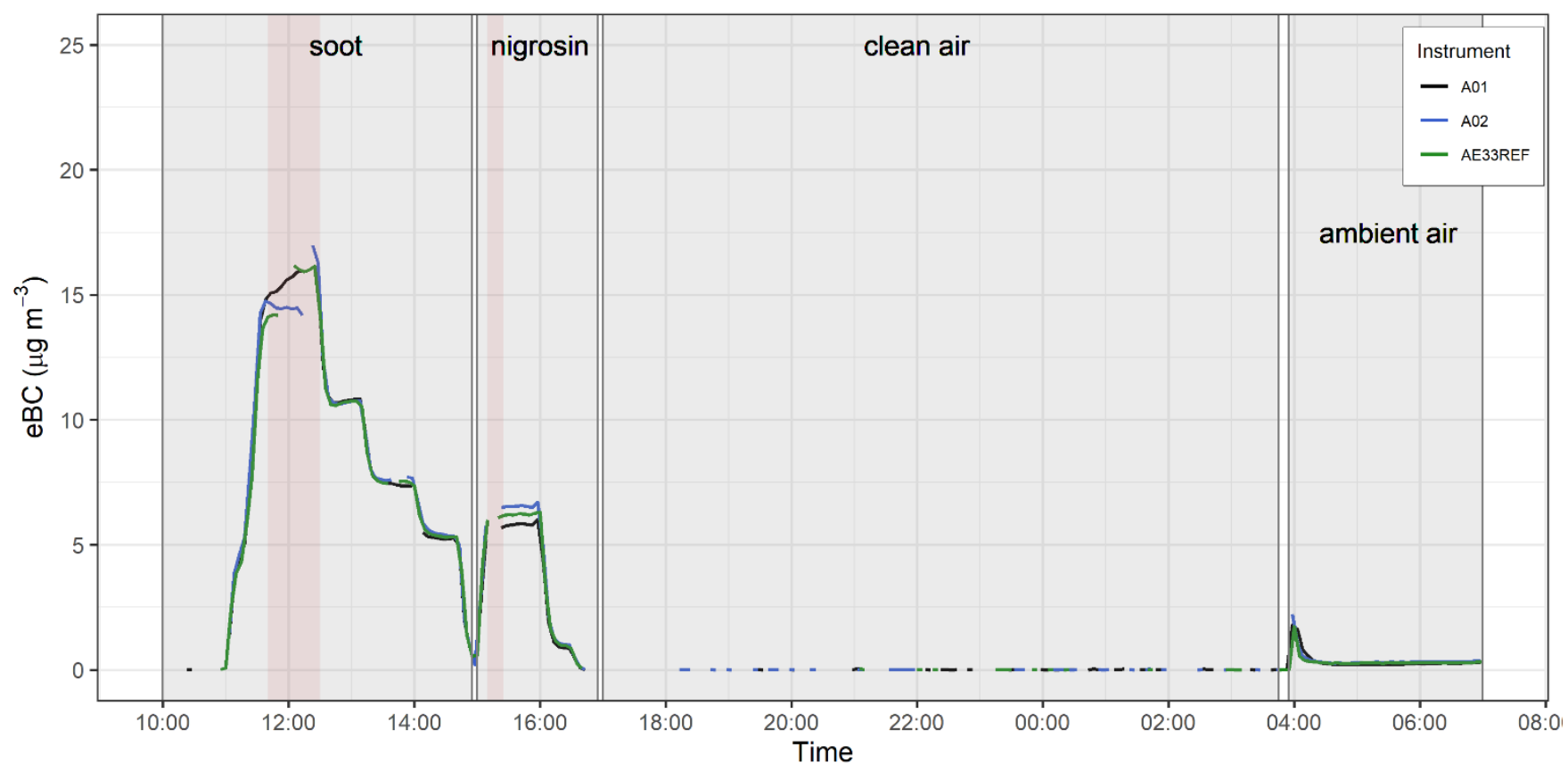

15 Figure S2. Time series of eBC mass concentrations at $880 \mathbf{~ n m}$ before maintenance in group A.

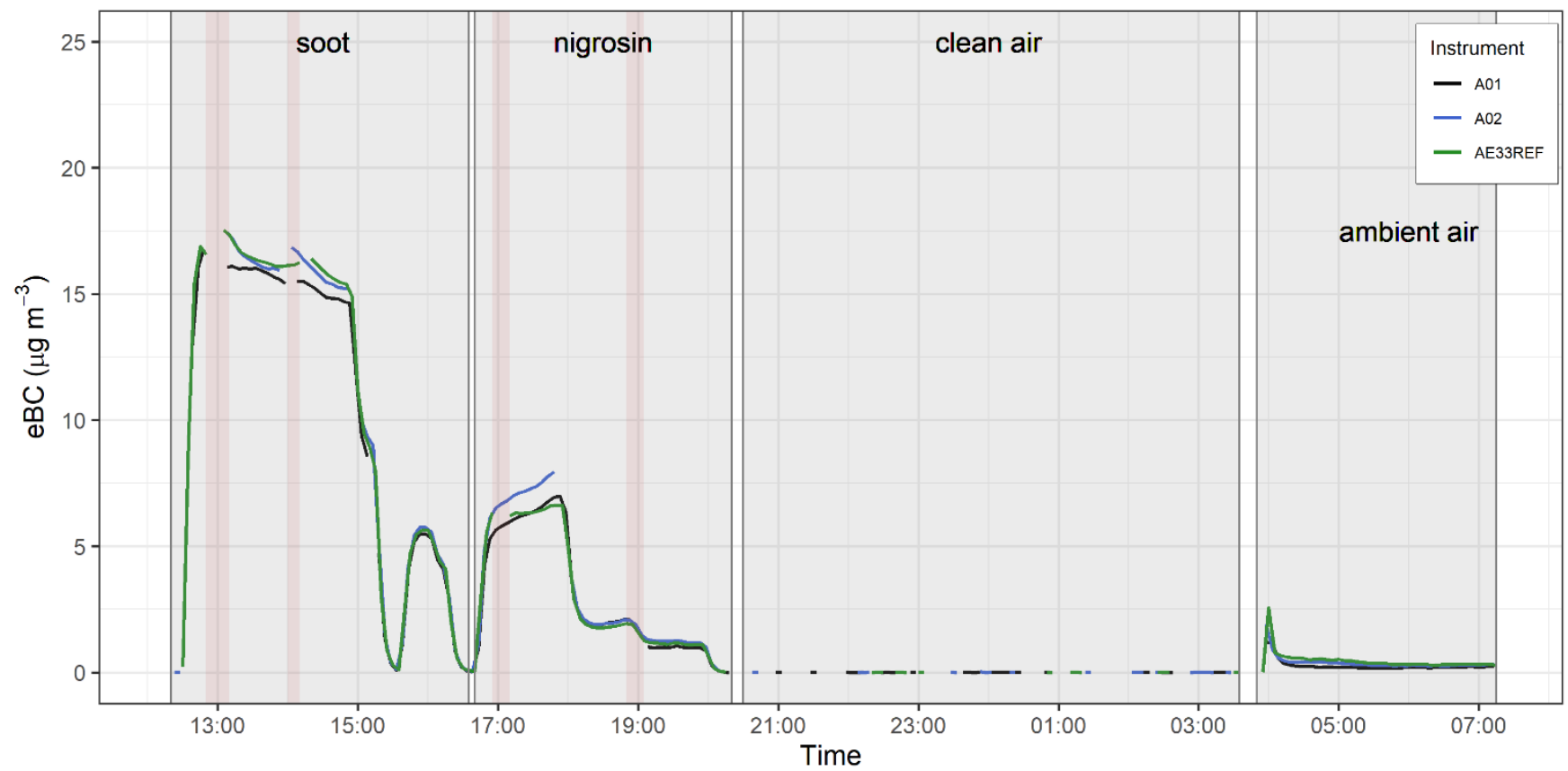

Figure S3. Time series of $\mathrm{eBC}$ mass concentrations at $880 \mathrm{~nm}$ after maintenance in group $\mathrm{A}$. 


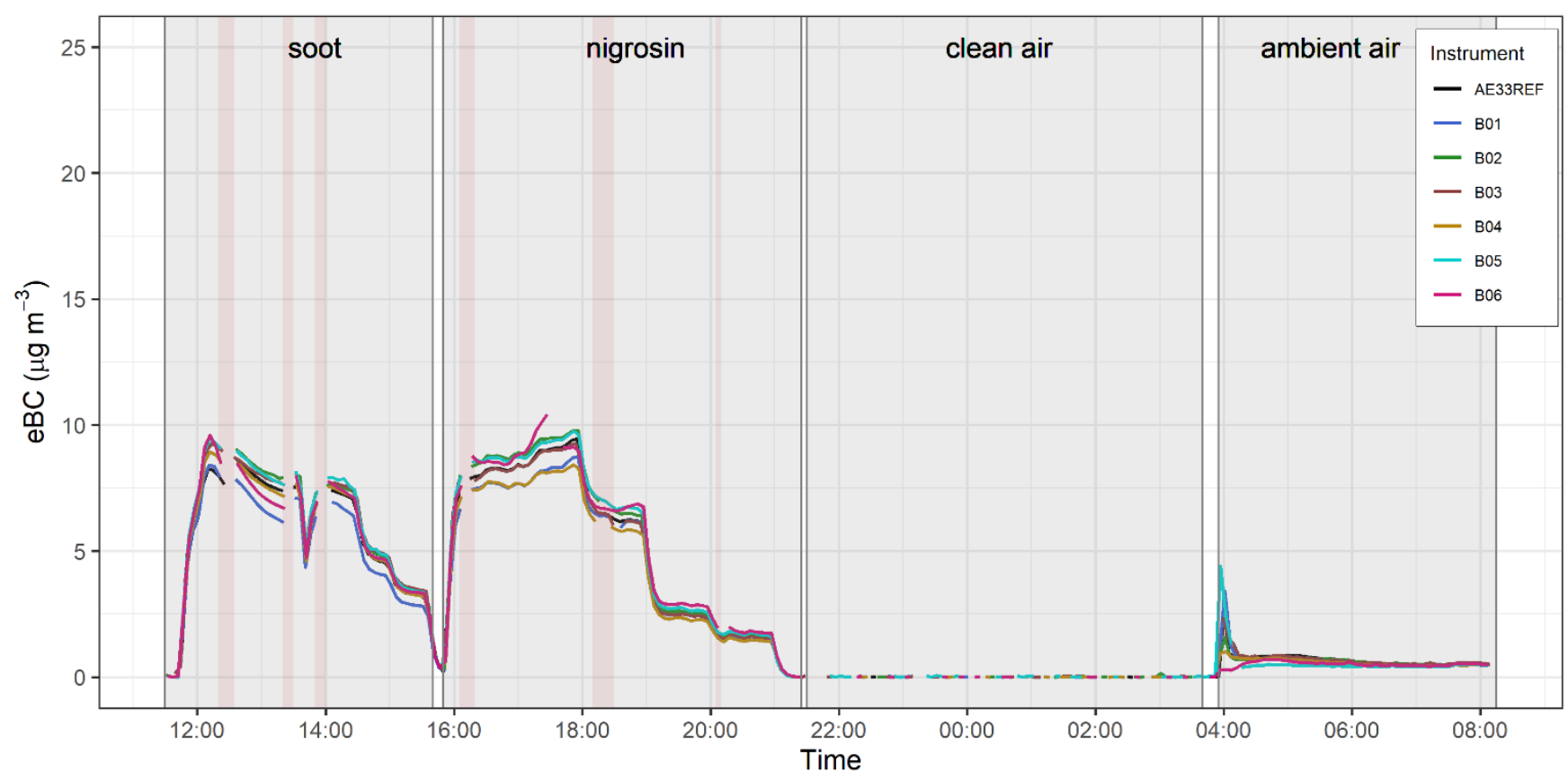

Figure S4. Time series of $\mathrm{eBC}$ mass concentrations at $880 \mathrm{~nm}$ before maintenance in group $\mathrm{B}$.

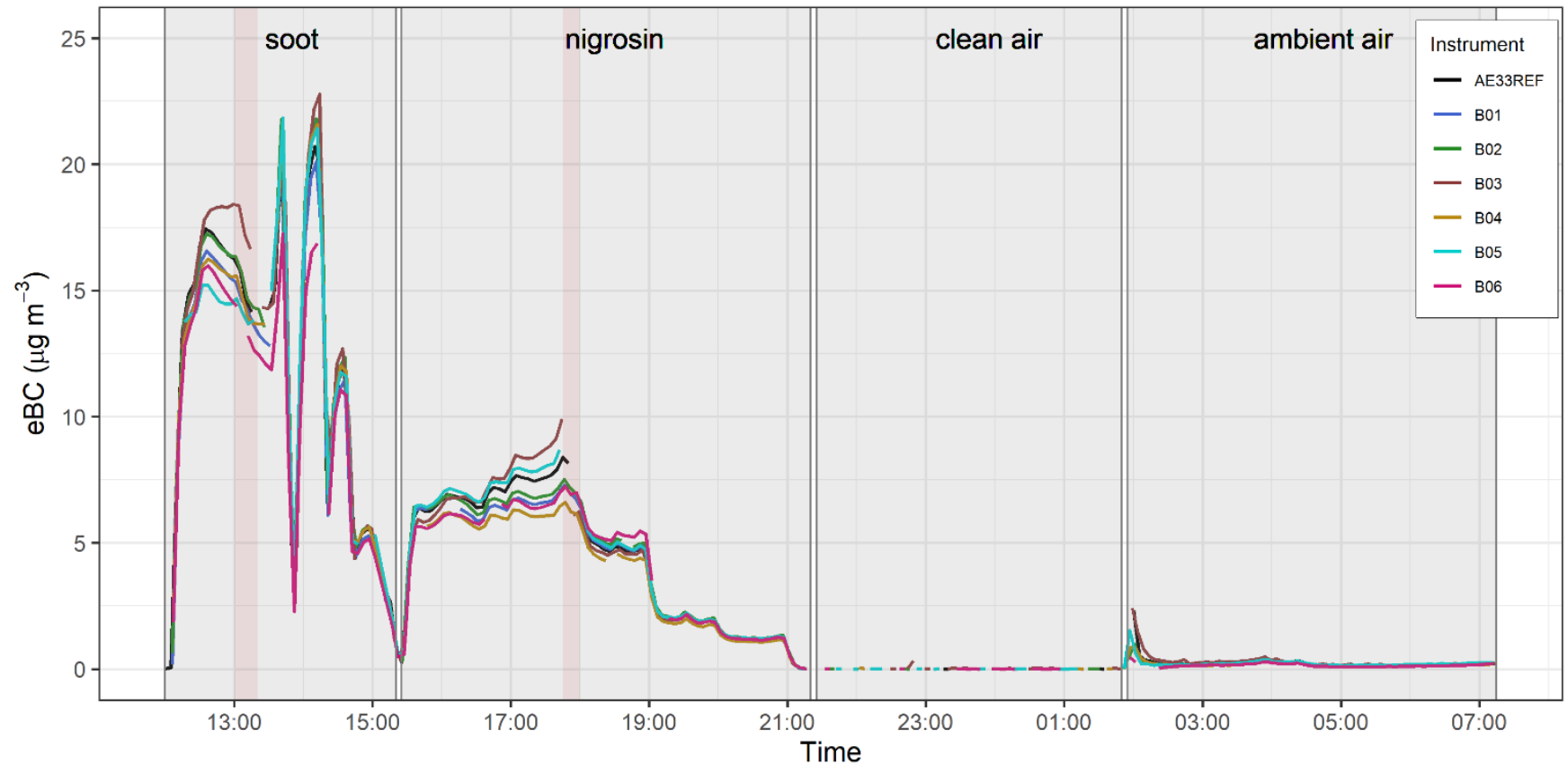

Figure S5. Time series of eBC mass concentrations at $880 \mathrm{~nm}$ after maintenance in group $\mathrm{B}$. 


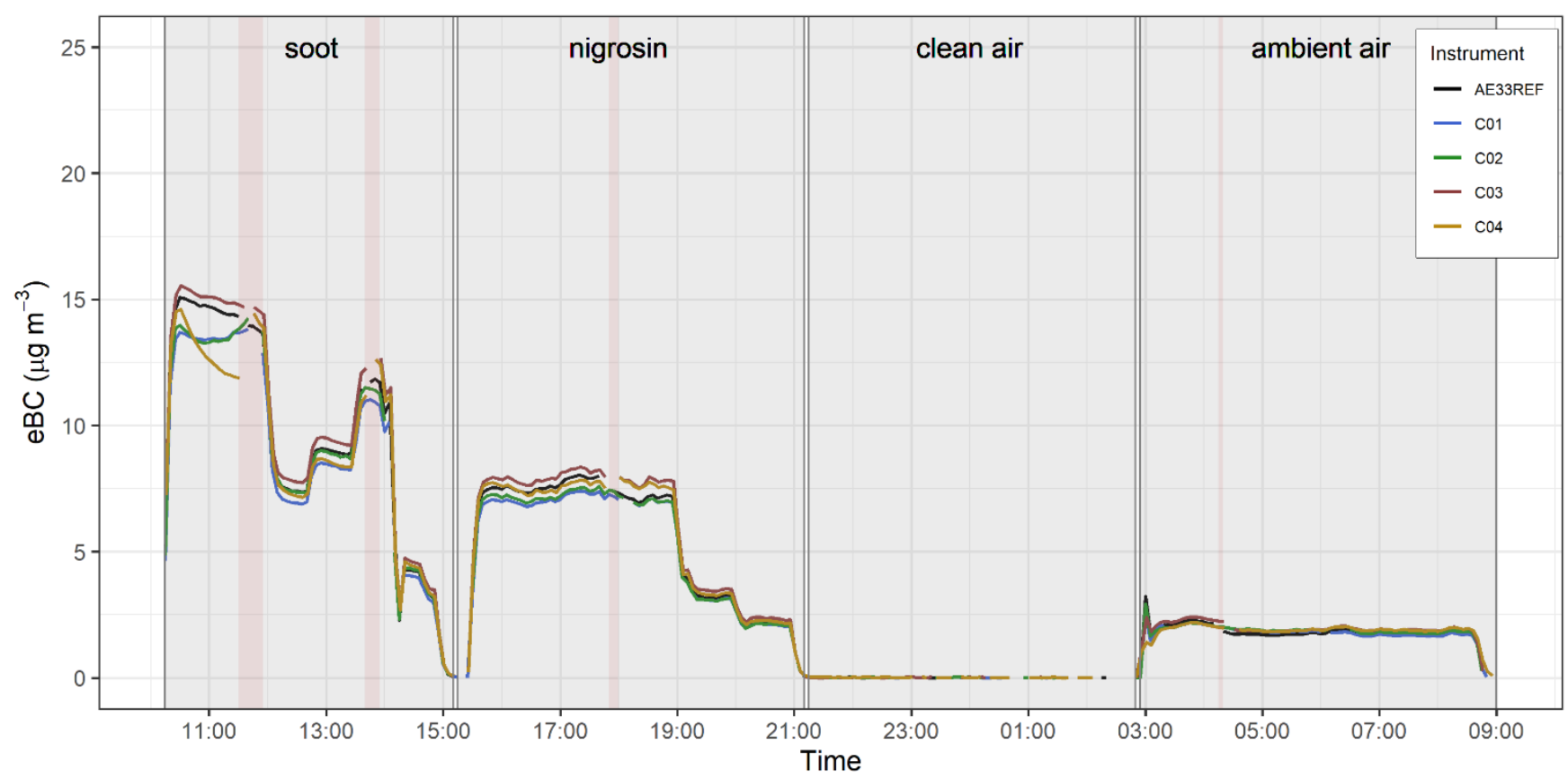

Figure S6. Time series of eBC mass concentrations at $880 \mathrm{~nm}$ before maintenance in group $\mathrm{C}$.

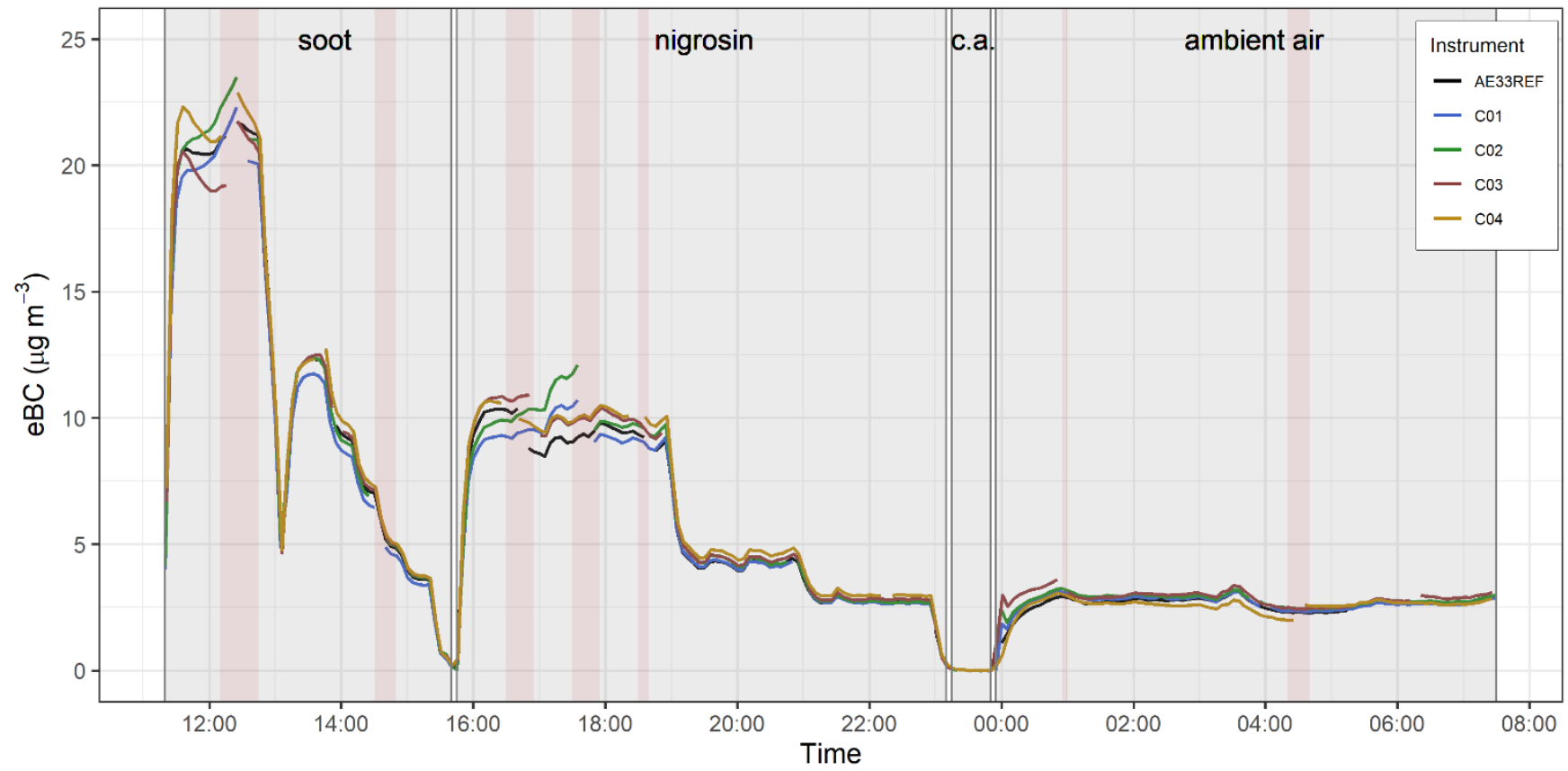

25 Figure S7. Time series of eBC mass concentrations at $880 \mathbf{~ n m}$ after maintenance in group C. c.a.: clean air. 


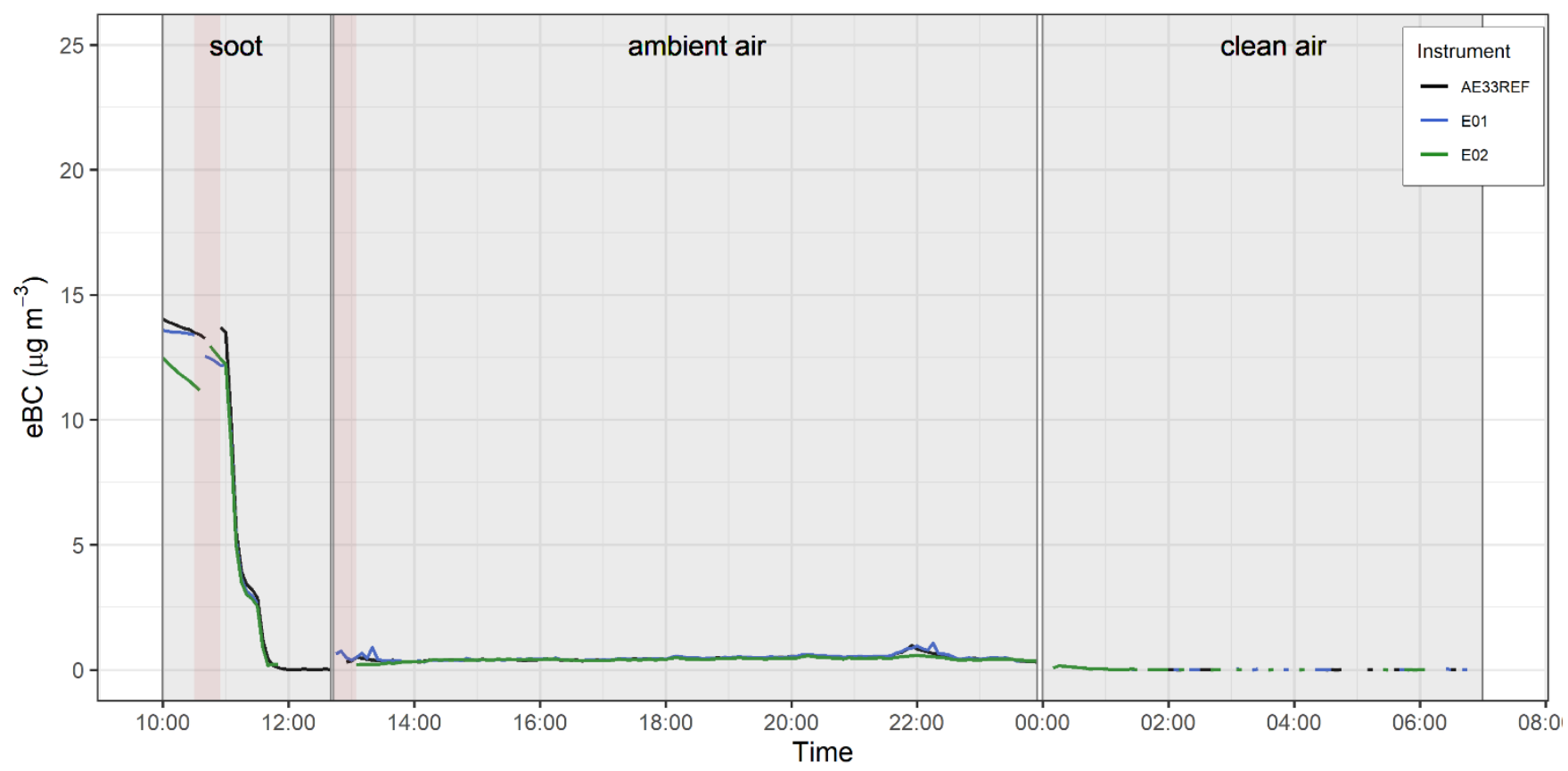

Figure S8. Time series of eBC mass concentrations at $880 \mathrm{~nm}$ before maintenance in group E. Note: Instruments from group $\mathrm{E}$ did not measure nigrosin particles.

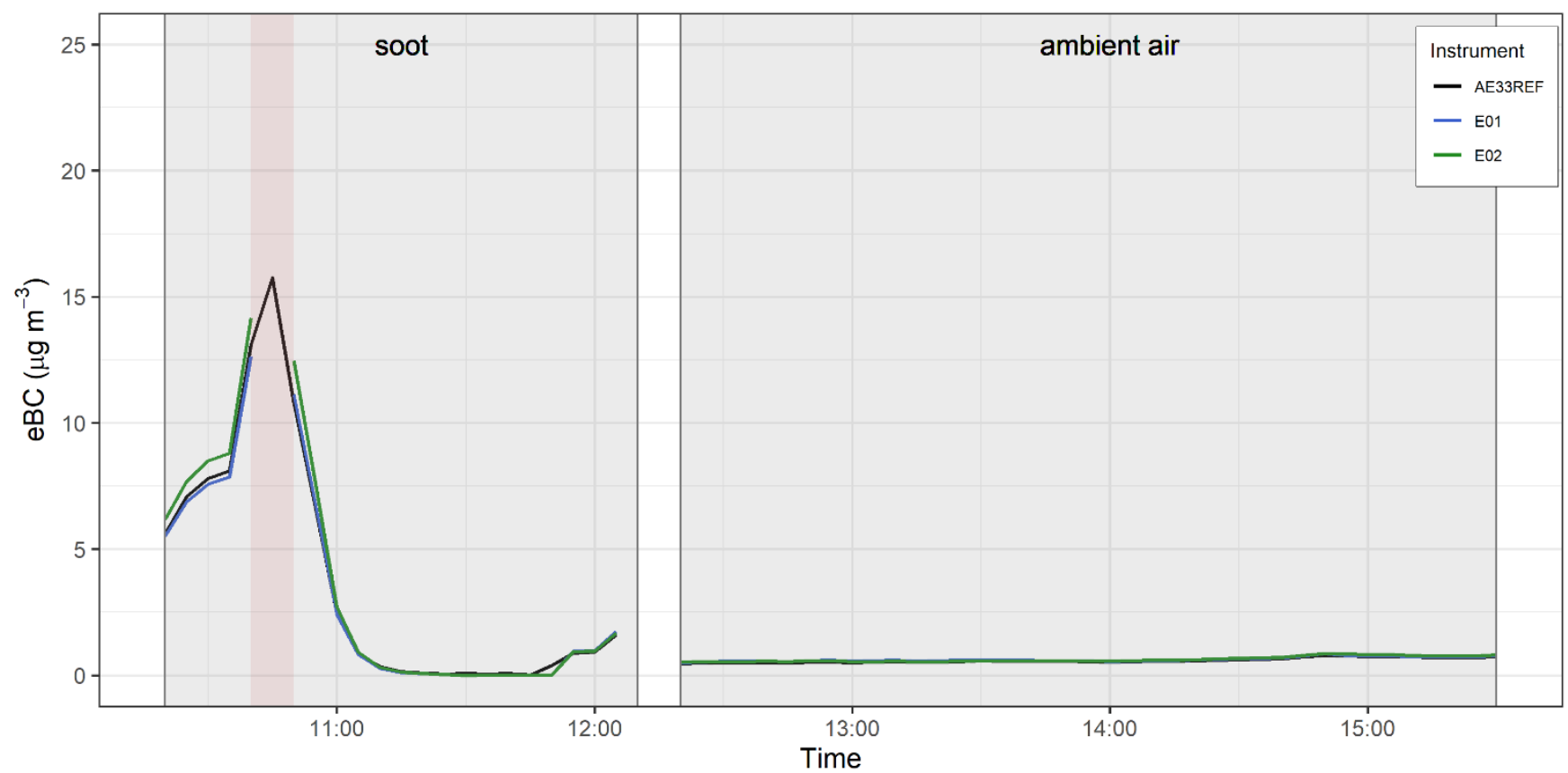

30 Figure S9. Time series of eBC mass concentrations at $880 \mathbf{~ n m}$ after maintenance in group E. Note: Instruments from group $\mathrm{E}$ did not measure nigrosin particles, neither clean air after maintenance. 


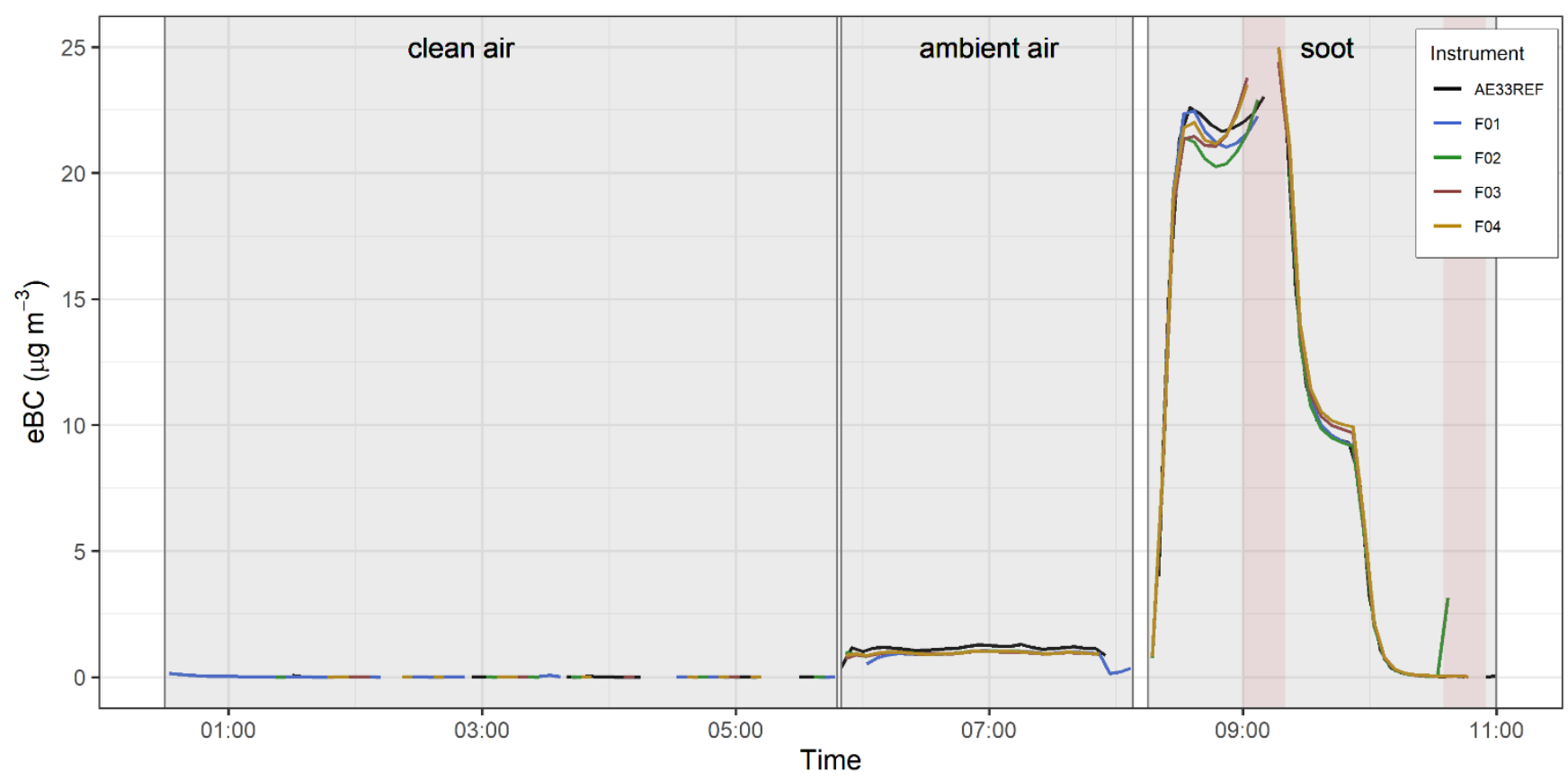

Figure S10. Time series of eBC mass concentrations at $880 \mathrm{~nm}$ before maintenance in group F. Note: the order of the samples was changed because of technical adjustments in the laboratory. Instruments from group F did not measure nigrosin particles.

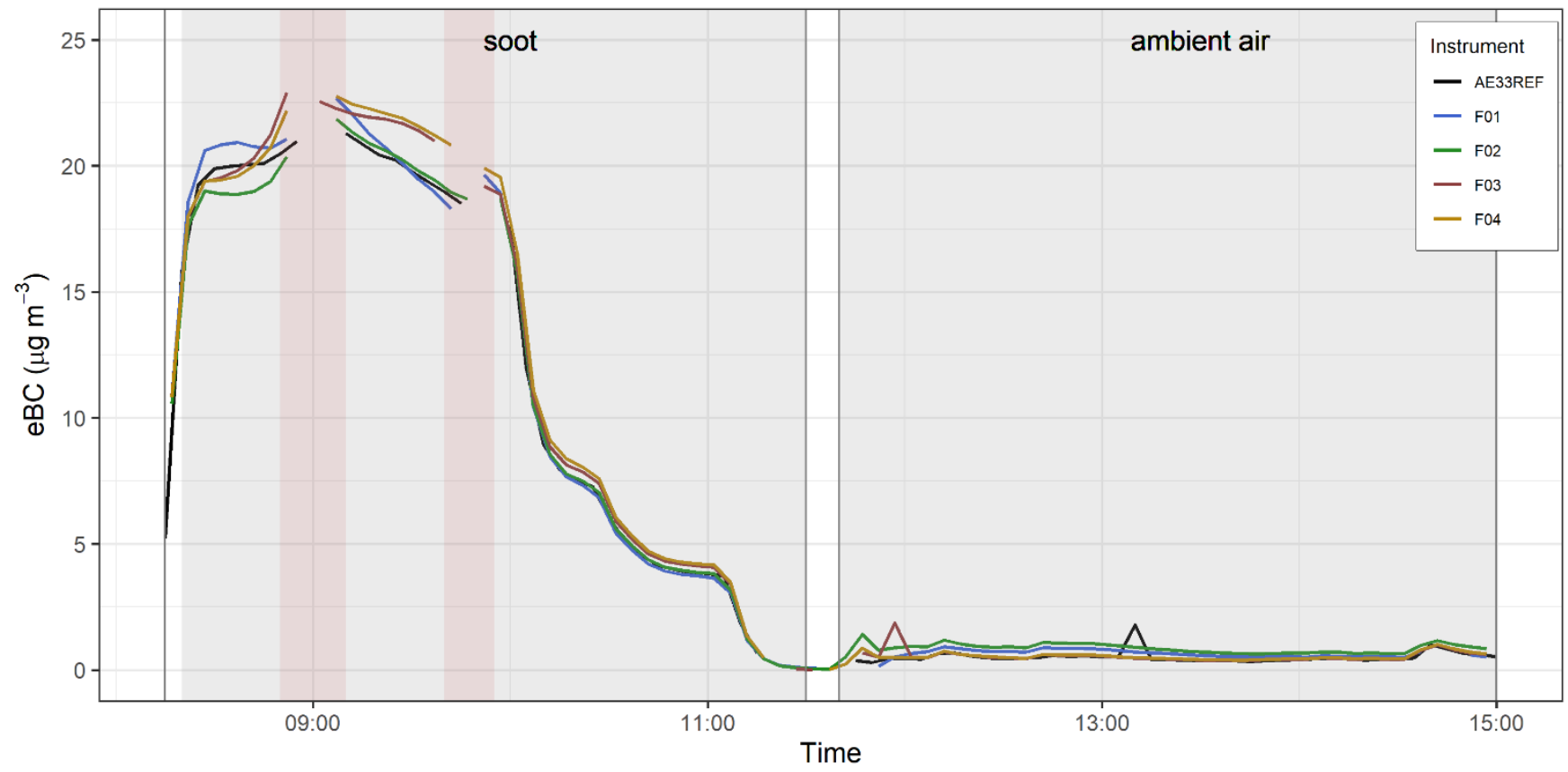

Figure S11. Time series of $\mathrm{eBC}$ mass concentrations at $880 \mathrm{~nm}$ after maintenance in group F. Note: Instruments from group $\mathrm{F}$ did not measure nigrosin particles, neither clean air after maintenance. 

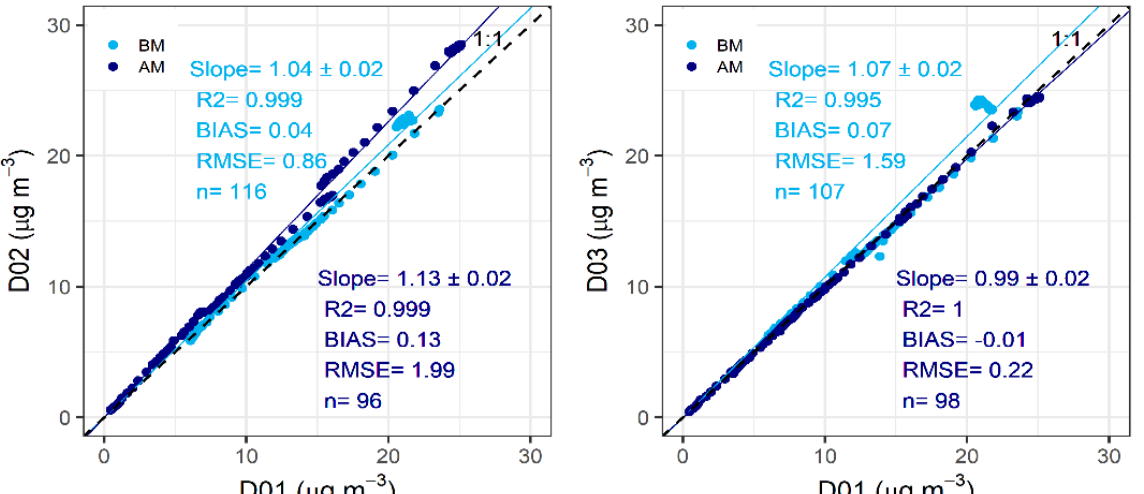

$\mathrm{D} 01\left(\mu \mathrm{g} \mathrm{m} \mathrm{m}^{-3}\right)$
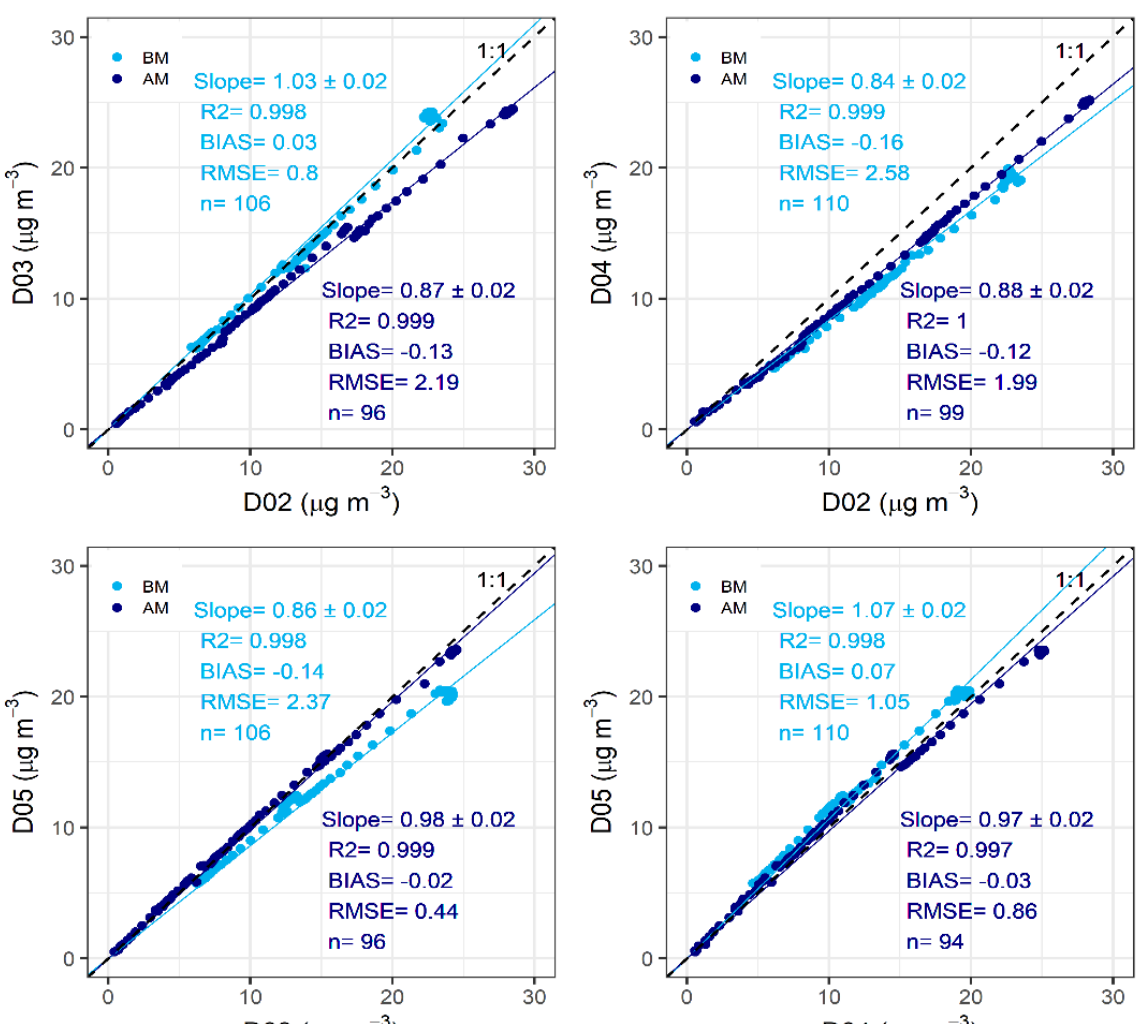

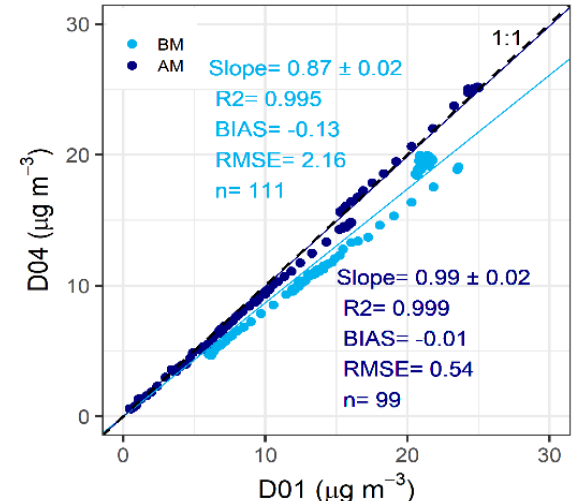

$\mathrm{D} 01\left(\mu \mathrm{g} \mathrm{m} \mathrm{m}^{-3}\right)$
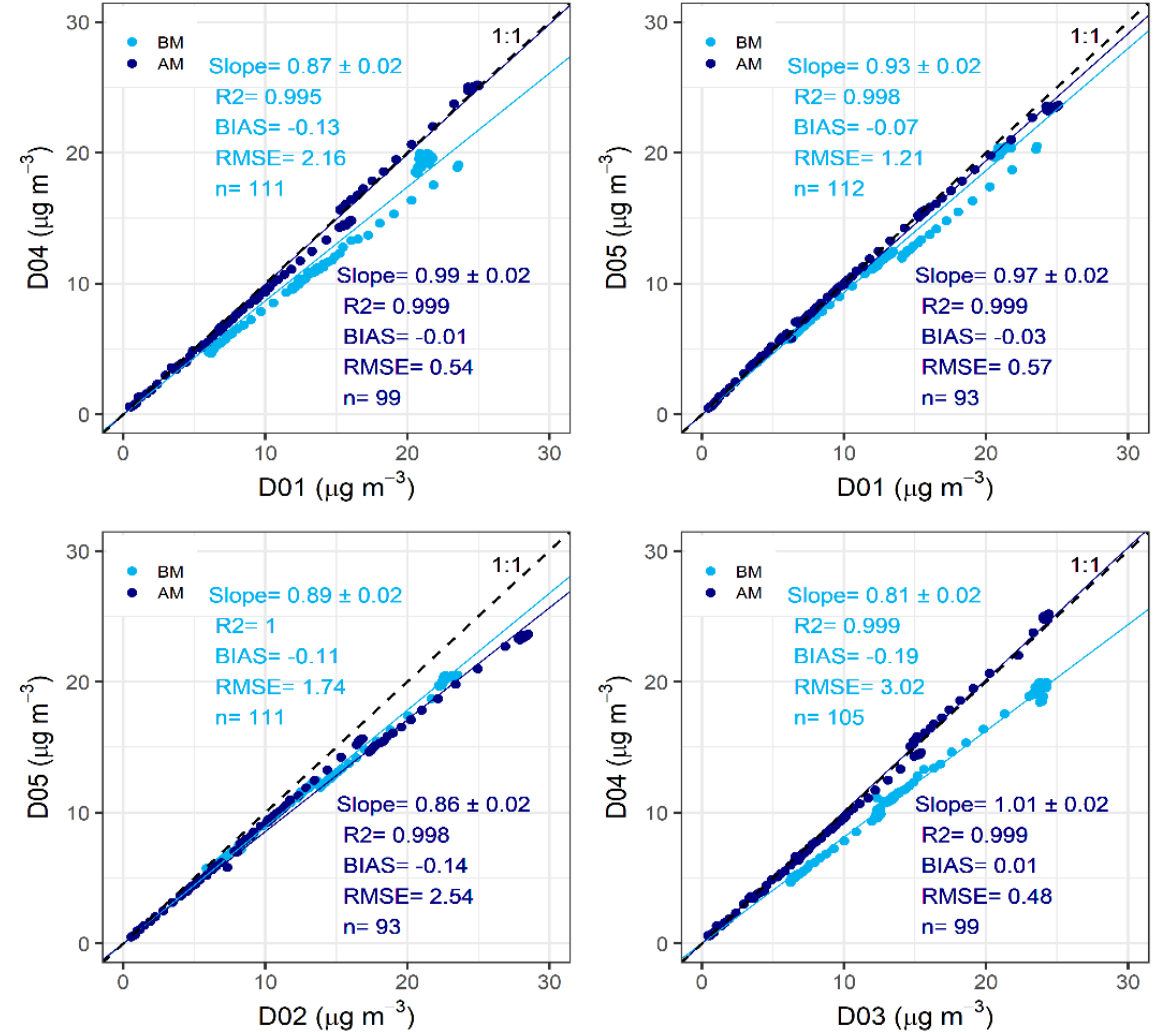

$\mathrm{D} 01\left(\mu \mathrm{g} \mathrm{m}^{-3}\right)$

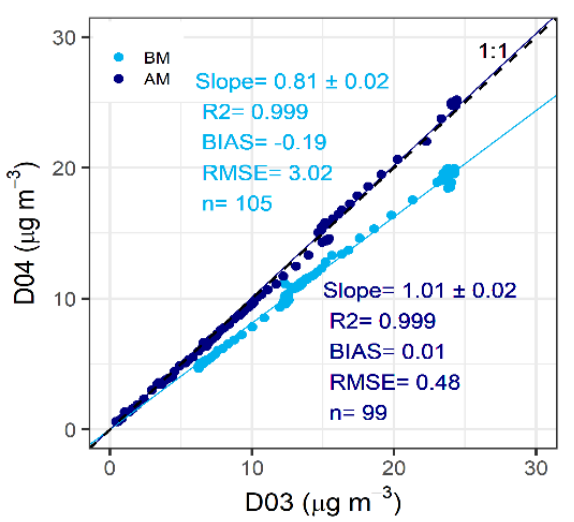

Figure S12. Scatter plot matrix for the comparison of measurements of $\mathrm{BC}$ at $880 \mathrm{~nm}$ from instruments in group $\mathrm{D}$, before and after maintenance during soot measurements. The intercept was forced through zero. BM: Before maintenance, AM: After maintenance. 

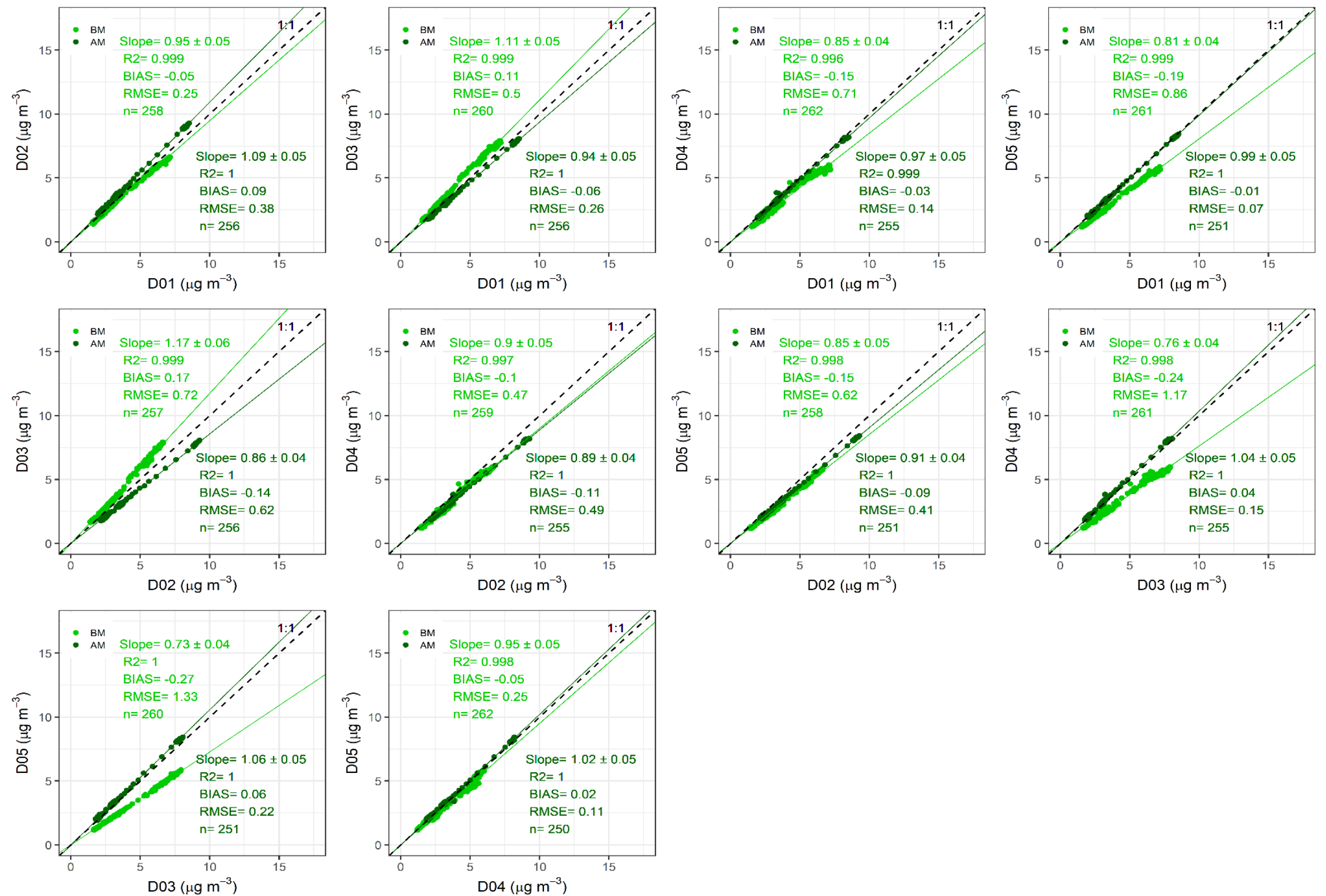

Figure S13. Scatter plot matrix for the comparison of measurements of $\mathrm{BC}$ at $880 \mathrm{~nm}$ from instruments in group $\mathrm{D}$, before and after maintenance during 


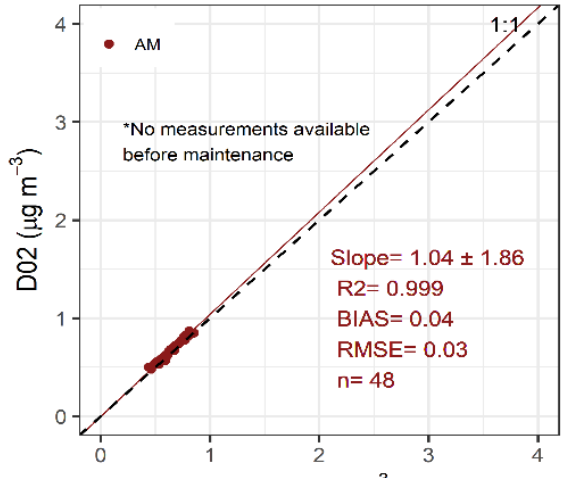

$\mathrm{D} 01\left(\mu \mathrm{g} \mathrm{m}^{-3}\right)$
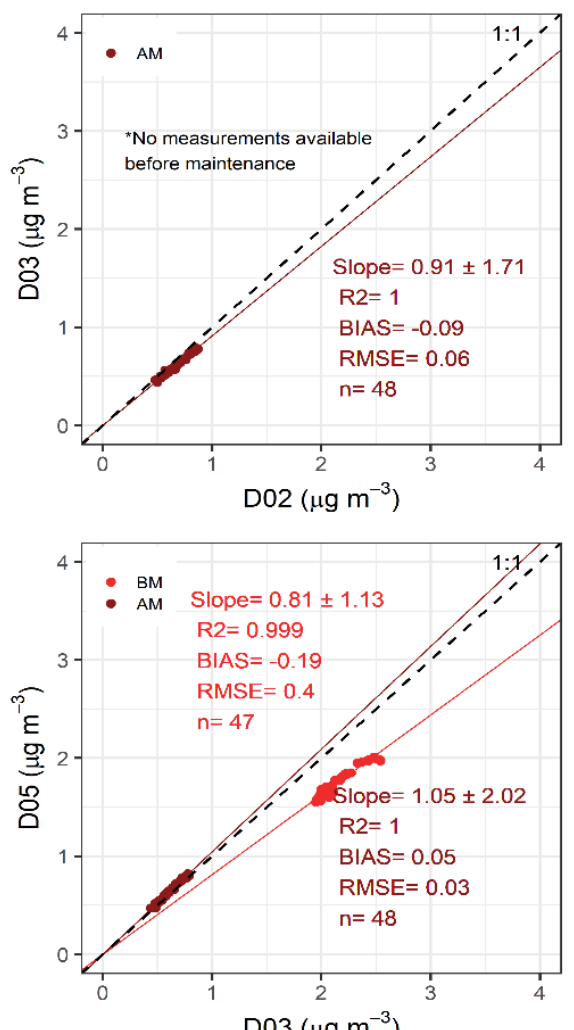

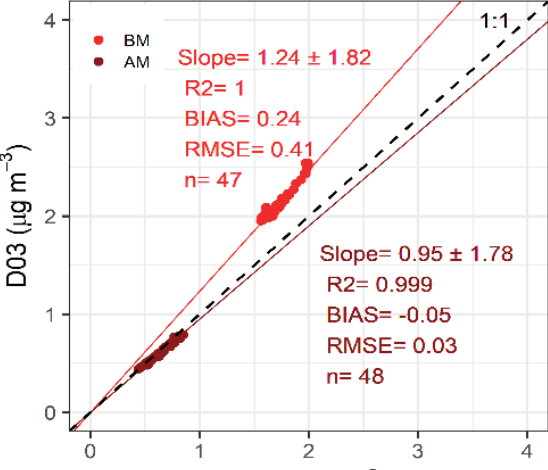

D01 $\left(\mu \mathrm{g} \mathrm{m}^{-3}\right)$
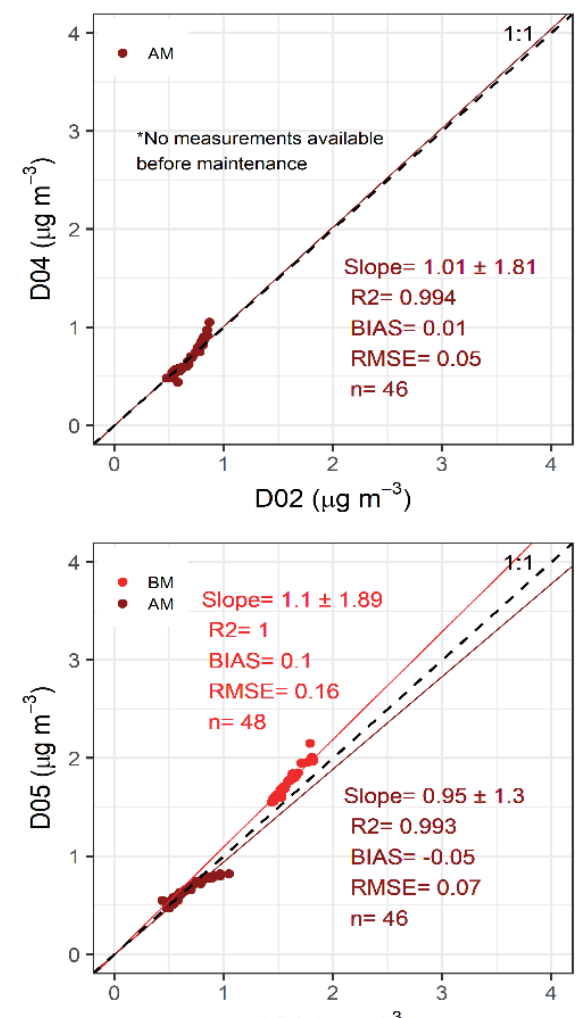

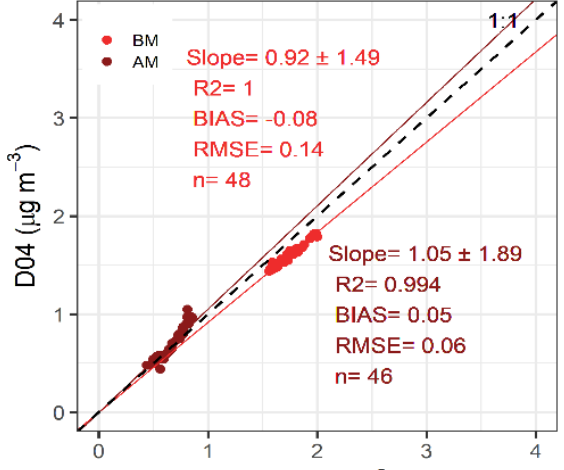

$\mathrm{D} 01\left(\mu \mathrm{g} \mathrm{m}^{-3}\right)$
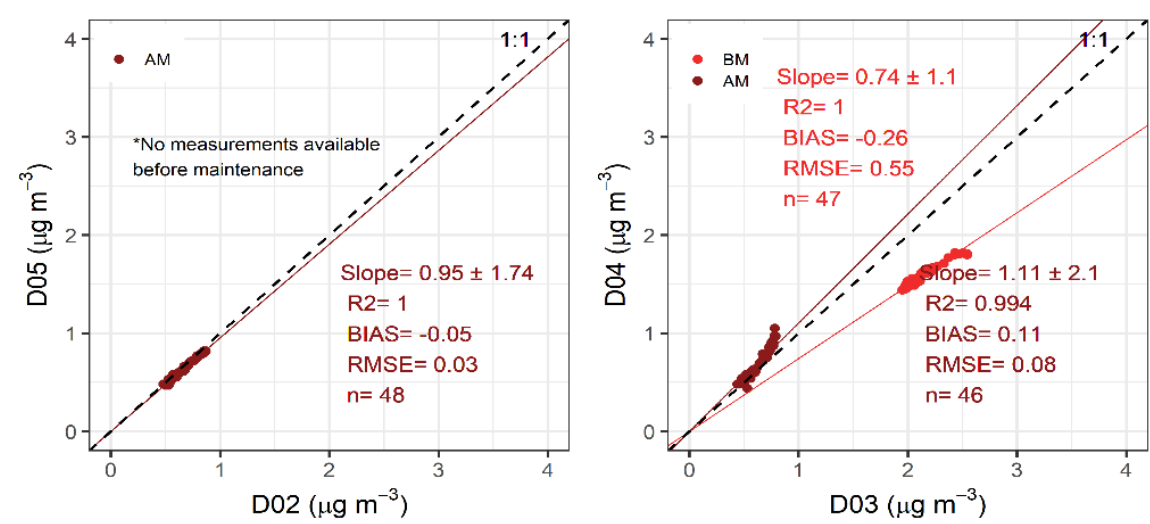

Figure S14. Scatter plot matrix for the comparison of measurements of $\mathrm{BC}$ at $880 \mathrm{~nm}$ from instruments in group $\mathrm{D}$, before and after maintenance during ambient air measurements. The intercept was forced through zero. BM: Before maintenance, AM: After maintenance. 
Table S1. Valid statuses of data used in the analysis.

\begin{tabular}{|c|l|}
\hline Status & \multicolumn{1}{|c|}{ Description } \\
\hline 0 & Measurement \\
\hline 8 & Check flow status history (warning) \\
\hline 128 & Tape warning (less than 30 spots left) \\
\hline 136 & Tape warning (less than 30 spots left) + check flow status history \\
\hline 256 & Tape last warning (less than 5 spots left) \\
\hline 65535 & $\begin{array}{l}\text { Database bigger than } 2 * 10^{6} \text { lines, i.e., a warning because the memory of the instrument } \\
\text { is getting full - the user has to make sure the data are recorded correctly, in some cases } \\
\text { data with this status are not valid because overwriting conflicts. }\end{array}$ \\
\hline
\end{tabular}

Table S2. Instrumental noise before and after maintenance.

\begin{tabular}{|c|c|c|c|c|c|c|c|c|c|c|c|c|c|c|}
\hline \multicolumn{15}{|c|}{ Noise $\left(\mu \mathrm{g} \mathrm{m}^{-3}\right)$} \\
\hline \multirow{2}{*}{ Instrument } & \multicolumn{2}{|c|}{ 370nm } & \multicolumn{2}{|c|}{ 470nm } & \multicolumn{2}{|c|}{ 520nm } & \multicolumn{2}{|c|}{$590 \mathrm{~nm}$} & \multicolumn{2}{|c|}{$660 \mathrm{~nm}$} & \multicolumn{2}{|c|}{ 880nm } & \multicolumn{2}{|c|}{ 950nm } \\
\hline & BM & $\mathbf{A M}$ & $\mathbf{B M}$ & $\mathbf{A M}$ & $\mathbf{B M}$ & $\mathbf{A M}$ & BM & $\mathbf{A M}$ & BM & $\mathbf{A M}$ & BM & $\mathbf{A M}$ & $\mathbf{B M}$ & $\mathbf{A M}$ \\
\hline \multicolumn{15}{|c|}{ Group A } \\
\hline A01 & 0.022 & 0.013 & 0.029 & 0.021 & 0.034 & 0.051 & 0.041 & 0.021 & 0.035 & 0.019 & 0.029 & 0.025 & 0.027 & 0.027 \\
\hline A02 & 0.015 & 0.013 & 0.022 & 0.017 & .023 & 0.02 & 0.035 & 0.024 & 0.025 & .021 & 0.025 & 0.024 & 0.027 & 0.022 \\
\hline \multicolumn{15}{|c|}{ Group B } \\
\hline B01 & 0.026 & ND & 0.035 & ND & 0.05 & ND & 0.038 & ND & 0.042 & ND & 0.026 & ND & 0.026 & ND \\
\hline B02 & 0.028 & 0.019 & 0.032 & 0.023 & 0.039 & 0.025 & 0.044 & 0.025 & 0.041 & 0.024 & 0.031 & 0.026 & 0.032 & 0.027 \\
\hline B03 & 0.033 & 0.043 & 0.052 & 0.061 & 0.059 & 0.074 & 0.057 & 0.071 & 0.062 & 0.071 & 0.063 & 0.062 & 0.05 & 0.058 \\
\hline B04 & 0.026 & 0.021 & 0.043 & 0.034 & 0.049 & 0.036 & 0.053 & 0.032 & 0.069 & 0.034 & 0.032 & 0.029 & 0.029 & 0.025 \\
\hline B05 & 0.099 & 0.02 & 0.141 & 0.033 & 0.161 & 0.039 & 0.176 & 0.042 & 0.134 & 0.035 & 0.068 & 0.03 & 0.053 & 0.029 \\
\hline B06 & 0.012 & 0.014 & 0.021 & 0.02 & 0.019 & 0.022 & 0.02 & 0.024 & 0.018 & 0.022 & 0.022 & 0.017 & 0.02 & 0.023 \\
\hline \multicolumn{15}{|c|}{ Group C } \\
\hline $\mathrm{C} 01$ & 0.02 & 0.013 & 0.03 & 0.027 & 0.029 & 0.016 & 0.037 & 0.026 & 0.032 & 0.015 & 0.026 & 0.017 & 0.025 & 0.016 \\
\hline $\mathrm{C} 02$ & 0.032 & 0.022 & 0.092 & 0.036 & 0.051 & 0.031 & 0.045 & 0.034 & 0.042 & 0.03 & 0.031 & 0.021 & 0.031 & 0.037 \\
\hline $\mathrm{C} 03$ & 0.038 & 0.046 & 0.053 & 0.057 & 0.053 & 0.064 & 0.062 & 0.062 & 0.051 & 0.063 & 0.037 & 0.038 & 0.038 & 0.037 \\
\hline $\mathrm{C} 04$ & 0.032 & 0.013 & 0.048 & 0.016 & 0.047 & 0.019 & 0.06 & 0.016 & 0.049 & 0.017 & 0.028 & 0.025 & 0.03 & 0.02 \\
\hline \multicolumn{15}{|c|}{ Group D } \\
\hline D01 & 0.022 & 0.013 & 0.024 & 0.017 & 0.028 & 0.017 & 0.03 & 0.028 & 0.029 & 0.025 & 0.032 & 0.034 & 0.038 & 0.036 \\
\hline D02 & 0.015 & 0.066 & 0.026 & 0.06 & 0.02 & 0.087 & 0.022 & 0.092 & 0.018 & 0.06 & 0.025 & 0.04 & 0.022 & 0.038 \\
\hline D03 & 0.055 & 0.014 & 0.058 & 0.021 & 0.073 & 0.016 & 0.074 & 0.021 & 0.17 & 0.029 & 0.042 & 0.044 & 0.043 & 0.048 \\
\hline D04 & 0.044 & 0.018 & 0.051 & 0.029 & 0.054 & 0.028 & 0.062 & 0.03 & 0.063 & 0.028 & 0.056 & 0.032 & 0.06 & 0.033 \\
\hline D05 & 0.073 & 0.034 & 0.068 & 0.045 & 0.069 & 0.05 & 0.052 & 0.046 & 0.056 & 0.038 & 0.035 & 0.035 & 0.031 & 0.041 \\
\hline \multicolumn{15}{|c|}{ Group E } \\
\hline E01 & 0.017 & ND & 0.034 & ND & 0.03 & ND & 0.026 & ND & 0.024 & ND & 0.022 & ND & 0.022 & ND \\
\hline E02 & 0.019 & ND & 0.019 & ND & 0.023 & ND & 0.023 & ND & 0.021 & ND & 0.023 & ND & 0.028 & ND \\
\hline \multicolumn{15}{|c|}{ Group F } \\
\hline F01 & 0.023 & ND & 0.023 & ND & 0.024 & ND & 0.027 & ND & 0.028 & ND & 0.031 & ND & 0.035 & ND \\
\hline F02 & 0.013 & ND & 0.013 & ND & 0.016 & ND & 0.017 & ND & 0.017 & ND & 0.021 & ND & 0.022 & ND \\
\hline F03 & 0.012 & ND & 0.013 & ND & 0.014 & ND & 0.017 & ND & 0.014 & ND & 0.019 & ND & 0.022 & ND \\
\hline F04 & 0.011 & ND & 0.012 & ND & 0.012 & ND & 0.014 & ND & 0.015 & ND & 0.022 & ND & 0.025 & ND \\
\hline
\end{tabular}

BM: Before maintenance; AM: After maintenance; ND: No data. 